\title{
Type A aortic dissection
}

\section{Background}

Aortic dissection is a life-threatening condition which may be rapidly fatal if not detected and treated promptly. The aorta is the main artery which branches off the heart and supplies oxygen-rich blood to the body's organs and tissues. The wall of the aorta consists of inner, middle and outer layers. Aortic dissection occurs when there is a tear in the inner layer, which allows blood to enter through the tear and fill up between the inner and middle layers, causing these layers to separate or 'dissect'. Type A dissection occurs when the tear develops in the ascending part of the aorta just as it branches off the heart, while Type B dissection involves the lower aorta. While Type A dissection is the more dangerous form, chances of survival are significantly improved with early detection and management.

\section{Risk factors}

Aortic dissection is more common in males and in individuals over the age of 60 . The most important modifiable risk factor is longstanding high blood pressure, which results in excessive stress on the inside wall of the aorta, predisposing it to tearing. Other important risk factors include the presence of fatty plaques, areas of weakened tissue and bulging in the aorta and defects of the aortic valve, such as a bicuspid valve. There are also a number of genetic conditions which predispose the aorta to dissection. These include connective tissue disorders such as Marfan syndrome and Ehlers-Danlos syndrome, where the structural support within the aortic wall is weakened.

\section{Signs and symptoms}

Individuals with an acute type A aortic dissection typically experience a sudden onset of severe chest pain, which may have a tearing quality and may spread to the neck, jaw or back. Individuals may also experience shortness of breath, loss of consciousness and symptoms similar to those from a stroke, such as sudden difficulty speaking, visual loss and weakness on one side of the body. It should be appreciated that aortic dissection may occasionally occur without any symptoms.

\section{Diagnosis}

Prompt diagnosis of this serious condition requires early imaging of the heart and aorta. Imaging techniques include computed tomography (CT) scanning, whereby a series of $\mathrm{X}$-rays are generated to produce detailed crosssectional images of the heart and aorta. This may require the use of an injected contrast agent. Other imaging techniques include magnetic resonance imaging (MRI), which uses a magnetic field to generate detailed images, and echocardiography, which uses ultrasound waves and a probe inserted into the oesophagus to generate images of the nearby heart and aorta. Echocardiography is particularly useful in patients with suspected type A dissection who may deteriorate rapidly.

\section{Management and prevention}

Once diagnosed, type A aortic dissection requires urgent surgery. This involves removal of the dissected portion of aorta and reconstruction of the aorta with a synthetic tube. This is most often carried out by open surgery. In cases where the aortic valve is damaged as well, a replacement valve may be placed within the synthetic tube used to repair the dissected aorta. In addition, some medications, such as beta blockers and nitroprusside, may be used to lower heart rate and blood pressure to prevent worsening of the dissection prior to surgery. Prevention involves measures taken to lower blood pressure and regular surveillance by imaging for those at increased risk of dissection in the future. For more information, please visit the following websites: http://www.mayoclinic.org/diseases-conditions/aorticdissection/basics/definition/con-20032930 http://www.mayoclinic.org/medical-professionals/clinicalupdates/cardiovascular/evolving-management-type-aaortic-dissection http://emedicine.medscape.com/article/2062452-overview

doi: 10.21037/acs.2016.05.04

\begin{tabular}{|c|c|}
\hline Section Writer: Christopher Harris & For specific information concerning your medical condition, \\
\hline Illustration Editor: Beth Croce & ACS suggests that you consult your physician. This page may be \\
\hline Section Editor: Christopher Cao & \\
\hline
\end{tabular}

